\title{
DESIGN AND PROTOTYPING OF THE AMD FOR THE ILC
}

\author{
Haitao Wang ${ }^{1,2}$, Wanming Liu ${ }^{1}$, Wei Gai ${ }^{1}$, Thomas Wong ${ }^{2}$, Alex Kanareykin ${ }^{3}$ \\ ${ }^{1}$ ANL, Argonne, IL 60439, U.S.A. \\ ${ }^{2}$ Illinois Institute of Technology, Chicago, IL 60616, U.S.A. \\ ${ }^{3}$ Euclid TechLabs, LLC, Solon, OH 44139, U.S.A.
}

\section{Abstract}

An Adiabatic Matching Device (AMD), which generates a tapered magnetic field with initial on-axis field up to 5 Tesla, is required in ILC positron capturing optics. A proposed scheme is to use a pulsed normal conducting flux concentrator to generate the strong magnetic field. By varying the inner shape of a flux concentrator, one can shape the on-axis magnetic field profile of the AMD. In this paper, we present an equivalent circuit model of a pulsed flux concentrator based on frequency domain analysis. We have constructed a prototype flux concentrator based on the circuit model, and experimental results are presented to validate the model. Using the equivalent circuit model, a flux concentrator based AMD is designed for the ILC positron matching. The beam capturing simulation results using the designed AMD are presented in this paper.

\section{INTRODUCTION}

The Adiabatic Matching Device (AMD) is a critical part for the International Linear Collider (ILC) positron source. The positron beam emerging from the target has broad energy spread and large divergence. The AMD, which generates a tapered magnetic field with the initial field up to 5 Tesla, can match the positrons to the input aperture of Positron Pre-accelerator (PPA) [1]-[3]. Since the AMD will be operating in a radiation-hard environment, a pulsed flux concentrator is a good candidate for such a device [4], contrary to the superconducting DC magnet.

Fig. 1 shows the cross-sectional views of a typical flux concentrator [5]. It consists of a primary coil surrounding a solid conducting core with a radial slot connecting the inner bore of the core to its outer surface. A time dependant current in the primary coil will induce magnetic flux in the core. Due to the radial slot, the induced current is forced to flow to the inner surface, thus generating stronger magnetic fields in the center. The direction of the current is shown in Fig 1. The induced current shifts the primary coil flux into the smaller region inside the central bore. In addition, a benefit of the flux concentrator structure is that the field profile can be changed by the shape of the taper.

In this paper we consider the coupling between the flux concentrator and external power supply circuits. A coupled equivalent circuit model is developed to model a flux concentrator. Such a circuit model has been

*Work supported by the US Department of Energy Office of Science under Contract No. DE-AC02-06CH11357. successfully applied to calculate the skin effect in transmission lines [6].
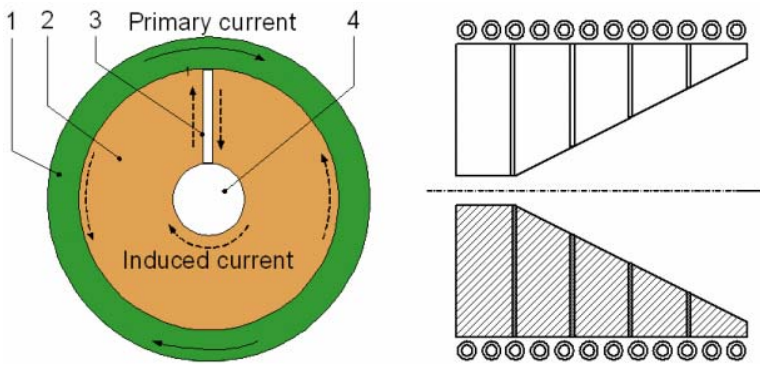

Figure 1: Cross-sectional view of a typical flux concentrator: 1 . primary coil, 2. central core, 3. radial slot, 4. bore.

In order to validate this model, we constructed a prototype flux concentrator. The measurement on the prototype structure and comparison with the calculation based on the model is presented in this paper. In addition, using the equivalent circuit model, a flux concentrator based AMD is designed for the ILC positron matching. The beam capturing simulation results using the designed AMD are also presented in this paper.

\section{CIRCUIT MODEL}

Modeling of the flux concentrator begins with dividing the central core into thin disks along the axial direction, followed by subdividing each disk into concentric rings. These rings are interconnected with each other at the slot location. We model each concentric ring as a circuit branch, including a resistor and an inductor that are serially connected together. The coupling between rings and rings to primary coil are done through mutual inductances. Any two adjacent rings within the same disks are connected by an equivalent resistor at the slot location to form a circuit loop. Modeling of the primary coil and the external electronics circuit follows the procedures in conventional representation of inductive circuits excited by switching voltage sources. The entire system consisting of the three parts is shown in Fig. 2.

Once a model for the equivalent coupled circuit network is established, the circuit parameters are calculated in a number of steps. First, the DC resistances of the concentric rings are calculated from their geometry and material properties. Then the self-inductance of each ring and the mutual inductance between rings and rings to the primary coil are evaluated using Neumann's formula [7]. With these circuit parameters, applying elementary 
circuit theory, a set of circuit equations can be derived from the coupled circuit network shown in Fig. 2. By solving these circuit equations, the frequency domain solution for the currents in the primary coil and all the concentric rings can be directly obtained. The calculation of the magnetic field at a reference location of the central axis for any single ring is straightforward for a given current. By synthesizing the effects from all the current rings of the central core and the primary coil, the frequency domain solution of the magnetic field at any given point are obtained and the transient response of the magnetic field can be found by inverse Fourier Transform.

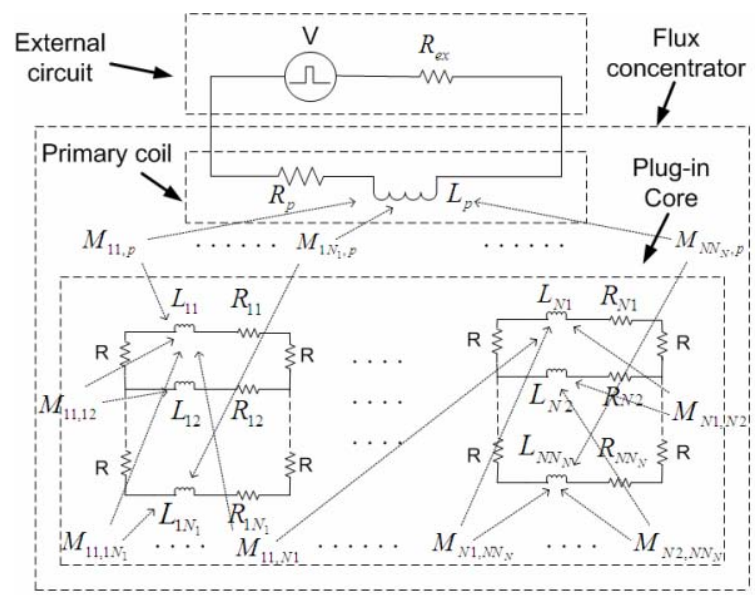

Figure 2: Modelling of entire flux concentrator.

\section{PROTOTYPE EXPERIMENT}

To assess the validity of the circuit model, a prototype flux concentrator was set up using an existing coil and a slotted aluminum core. Fig. 3 shows the schematic plot and a picture of the actual prototype flux concentrator. As an important part of the prototype system, a pulsed power supply was designed and built by integrating an IGBT module with a regulated DC power supply.
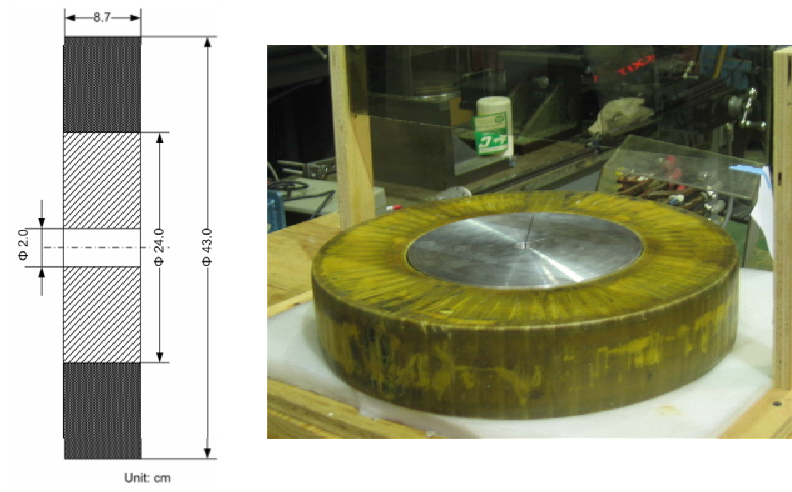

Figure 3: Schematic plot and picture of a prototype flux concentrator.

The transient responses of the voltage at the coil terminals, the current flowing through the coil, and the magnetic field at the central axis of the coil assembly are all monitored. Fig. 4 shows the measurement results for the prototype flux concentrator excited with voltage pulse of 50ms width from the pulsed power source.

With the same dimensions and material properties of the prototype structure, we calculated the transient response of the magnetic field using the circuit model developed in the previous session. Fig. 5 shows the comparison of the measurement data with the modeling results, which shows a good agreement. This gives us confidence for a design for the ILC AMD.
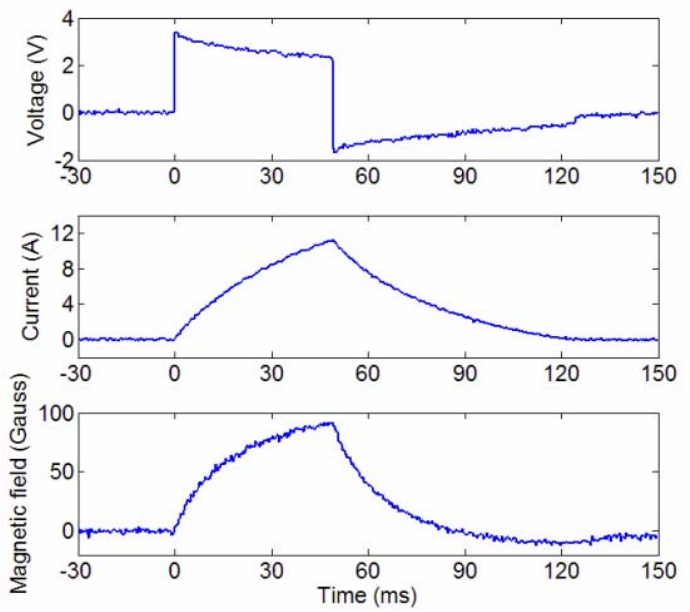

Figure 4: Transient responses of voltage, current and magnetic field of the prototype flux concentrator.

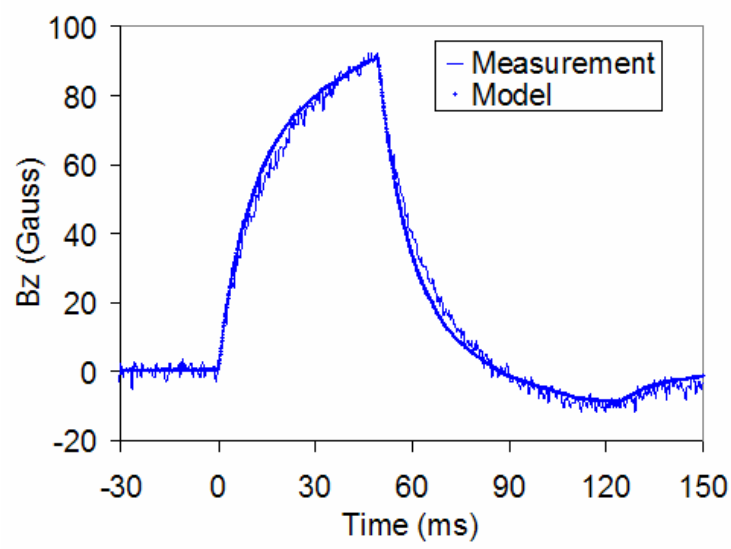

Figure 5: Comparison of the measured magnetic field with the modelling results of the flux concentrator.

\section{CONCEPTUAL AMD DESIGN FOR THE ILC}

In this section, we present a design of a flux concentrator for the ILC AMD using the circuit model. The AMD has the following parameters: magnetic field on the target exit 5 Tesla, pulse width $5 \mathrm{~ms}$, and repetition rate $5 \mathrm{~Hz}$. As shown in Fig 6, the AMD is comprised of two parts, one is a flux concentrator with excitation coil, operating at pulsed mode, and the other part is a tapered coil, operating at DC mode. The flux concentrator is cooled by liquid nitrogen $\left(\mathrm{T}=78^{\circ} \mathrm{K}\right)$. The $\mathrm{DC}$ coil operates 
at room temperature $\left(\mathrm{T}=293^{\circ} \mathrm{K}\right)$, and produces tapered magnetic field.

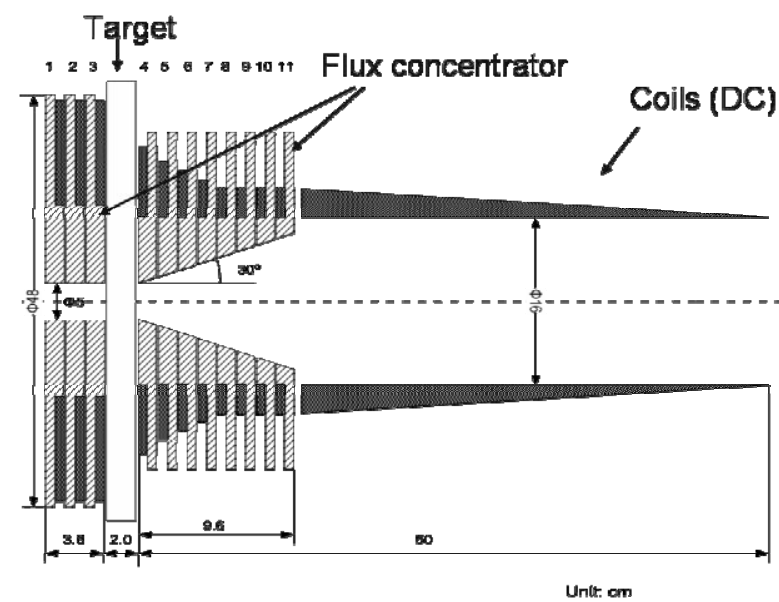

Figure: 6 Schematic layout of a flux concentrator based AMD for the ILC.
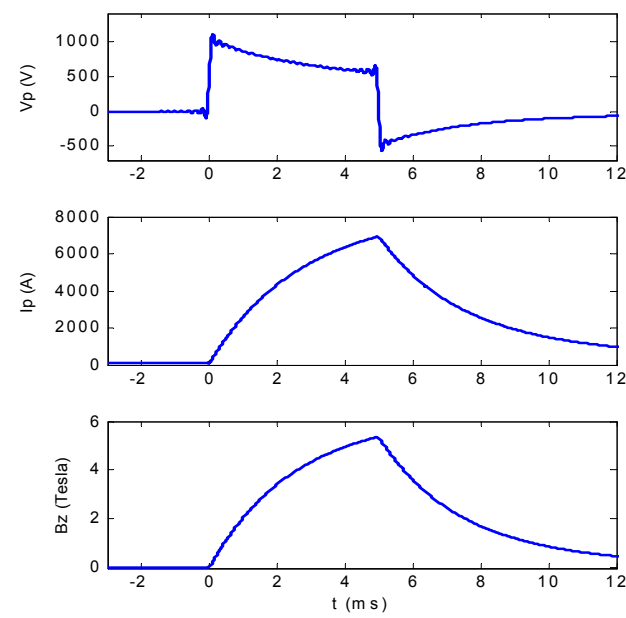

Figure: 7 The transient response of the flux concentrator for the ILC AMD.

Fig. 7 shows the transient response of the flux concentrator. The field profile along central axis is shown in Fig. 8. The peak power input to the AMD is around $5 \mathrm{MW}$, and the average power is about $200 \mathrm{KW}$.

As shown in Fig. 7, it is difficult to achieve absolute flattop pulse for more than $1 \mathrm{~ms}$ pulsed magnetic field for the designed flux concentrator. To investigate the effect of such varying field, magnetic field distributions at different time instants near the peak of the pulse are applied to the end to end positron simulation. The results of positron yield and polarization are compared. The field on target varies by about $8 \%$ at $1 \mathrm{~ms}$ range near the peak of pulse, but the yield varies less than $2 \%$ and the polarization does not change. According to the result, this design may work depending on the detailed ILC beam requirement.

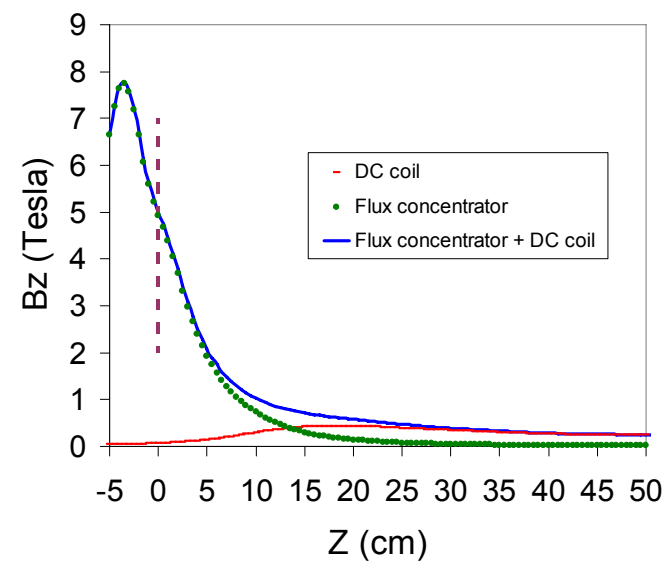

Figure: 8 Distribution of the longitudinal magnetic field along central axis, which is calculated at $4 \mathrm{~ms}$ after a pulse is applied.

\section{CONCLUSION}

A circuit model based on frequency domain analysis has been proposed for calculating the transient response of a flux concentrator. To validate the model, a prototype flux concentrator was designed and measured. The transient magnetic fields calculated by the circuit model showed a good agreement with the measured data.

Further a flux concentrator based AMD for the ILC positron source has been designed using the circuit model. The AMD has peak input power around 5MW, and average power about $200 \mathrm{KW}$.

\section{REFERENCES}

[1] http://www.astec.ac.uk/id_mag/BCD/Positron_Sourc e Description.doc.

[2] H. Wang, W. Liu, W. Gai, and K-J Kim, "Positron Emulator for Commissioning ILC Positron Source", Proc. of Particle Accelerator Conf., Knoxville, pp.2123-2125, 2005.

[3] Bharadwaj, Y. Batygin, R. Pitthan, J. Sheppard, H. Vincke, J. W. Wang, J. Gronberg, W. Stein. Proc. of Particle Accelerator Conf., Knoxville, pp. 3230-3232, 2005.

[4] Kulikov, S. D. Ecklund, and E. M. Reuter, "SLC Positron Source Pulsed Flux Concentrator", SLACPUB-5473, Proc. Of Particle Accelerator Conf., San Francisco, 1991.

[5] H. Brechna, D. A. Hill and B. M. Bally, "150 kOe Liquid Nitrogen Cooled Flux-Concentrator Magnet", Rev. Sci. Instr., 36 1529, 1965.

[6] W. T. Weeks, L. L. Wu, M.F. McAllister, and A. Singh, "Resistive and Inductive Skin Effect in Rectangular Conductor", IBM J. Res. Develop. Vol. 23, No. 6, 1979.

[7] S. Ramo, J. R. Whinnery, and T.V. Duzer, "Fields and Waves in Communication Electronics", John Wiley \& Sons, Inc., 1965. 